\title{
Protection of in-vitro grown Arabidopsis seedlings against abiotic stresses
}

\author{
Geert-Jan de Klerk · Paweena Pumisutapon
}

Received: 12 May 2008/Accepted: 8 July 2008/Published online: 22 July 2008

(C) The Author(s) 2008

\begin{abstract}
Severe abiotic stresses may cause permanent damage leading to death. In Arabidopsis seedlings germinating in vitro, we examined whether stress-related damage could be reduced by addition of protective low-molecular-weight compounds (trehalose and putrescine), addition of a specific signal molecule (acetylsalicylic acid), culture in the dark before and/or after the stress, and hardening mildstress pretreatments. All four tested protective procedures increased survival after exposure to drought, warm air, warm water or salinity, often from ca. $15 \%$ in the control to ca. $85 \%$ in the treated seedlings. These protective measures may be usable to reduce the negative effects of abiotic stresses related to tissue culture.
\end{abstract}

Keywords Heat stress - Drought stress · Priming · Salinity · Trehalose $\cdot$ Hot-water treatment . Hot-air treatment

\section{Abbreviations \\ HWT Hot-water treatment \\ HAT Hot-air treatment \\ MS Murashige-Skoog}

G.-J. de Klerk · P. Pumisutapon ( $₫)$

Wageningen Tissue Culture Centre, WUR-Plant

Breeding, P.O. Box 16, 6700 AA Wageningen,

The Netherlands

e-mail: paweena.pumisutapon@wur.nl

\author{
LEA Late-embryogenesis abundant \\ HSPs Heat shock proteins \\ ROS Reactive oxygen species
}

\section{Introduction}

Plants have developed a range of mechanisms to overcome damage by adverse environmental conditions. To survive predictable climatic stresses such as hot dry summers or cold winters, plants generate survival organs with specific morphological and biochemical properties, in particular dormant seeds and buds. To overcome other stresses, plants synthesize protective low-molecular-weight compounds, viz., proline, glycine betaine, polyamines or trehalose, and protective proteins, viz., late-embryogenesis abundant proteins (LEA-proteins; unknown function, Tunnacliffe and Wise 2007) and heat shock proteins (HSPs; chaperone-function, Wang et al. 2003). Since under severe stress conditions, the balance between the production of reactive oxygen species (ROS) and the antioxidant defense system is disturbed, increased antioxidant activity also occurs in stressed plants (review in Wang et al. 2003).

To obtain genotypes with improved stress tolerance, plants have been transformed with genes that confer the synthesis of protectants (trehalose, Garg et al. 2002; polyamines, Kasukabe et al. 2004), protective proteins (HSPs, Malik et al. 1999; LEAproteins, RoyChoudhury et al. 2007) and antioxidative 
enzymes (McKersie et al. 1999). Plants may be rendered resistant to severe stress in several other ways. Direct application of protectants results in resistance to stress (de Klerk 2002; Bae et al. 2005; Zeid and Shedeed 2006). Addition of signal molecules that form part of the signal transduction pathway for stress, may bring about protection (Lopez-Delgado et al. 1998). A foregoing moderate stress may harden against severe stress because the moderate stress sets off protective mechanisms (Larkindale et al. 2005). Finally, since photosynthesis is a major source of ROS, culture in darkness may also reduce the negative effects of stress.

Tissue-cultured plants are exposed to stress when they are acclimatized to ex-vitro conditions (de Klerk and Wijnhoven 2005) and during various techniques such as protoplast preparation, thermotherapy etc. Moreover, it has been suggested that several "unnatural" conditions inherent in tissue culture, e.g., mixotrophy, high levels of plant growth regulators and very high relative humidity (close to $100 \%$, Chen 2004) also impose major stresses in sensitive genotypes and cause typical tissue-culture problems such as hyperhydricity, recalcitrance and variation (review in de Klerk 2007). Since often cross-protection occurs (Sabehat et al. 1998), damage by typical in-vitro stresses may be reduced by treatments that confer resistance to other abiotic stresses. Thus, the protective measures mentioned above may reduce these tissueculture problems. In this article, we explore the effectiveness of various protective treatments in Arabidopsis seedlings germinating in vitro and exposed to common stresses (drought, heat and salinity).

\section{Materials and methods}

Plant material and growth conditions

Arabidopsis ecotype Columbia (Lehle Seeds, Roundrock, TX) was used. Seeds were surface-sterilized for 5 min in $70 \%$ ethanol followed by $10 \mathrm{~min}$ in $1.5 \%$ (w/v) hypochlorite $+0.02 \%$ Tween 20 . They were rinsed 5 times with water, dried and stored under sterile conditions at $4{ }^{\circ} \mathrm{C}$. Seeds were sown in a $60-\mathrm{mm}$ Petri dish with $8 \mathrm{ml}$ nutrient medium composed of $1 / 2 \mathrm{MS}$ (Murashige and Skoog 1962), 1.5\% sucrose and 0.7\% (w/v) agar (micro-agar, Duchefa, Haarlem, Netherlands), vernalized at $4{ }^{\circ} \mathrm{C}$ for 2 day in the dark to synchronize growth, and then kept at $20^{\circ} \mathrm{C}$ and a $16 \mathrm{~h}$ photoperiod (30 $\mu \mathrm{E} \mathrm{m}^{-2} \mathrm{~s}^{-1}$, Philips TL 33). Per Petri dish, 20 seeds were germinated. The germination percentage was close to 100 . The seeds were sown on a nylon gauze $(3.5 \times 3.5 \mathrm{~cm})$ on the medium. For transfer, e.g., for the drought treatment, the gauze with the seedlings was transferred. To remove excess of liquid, the gauze was kept for $5 \mathrm{~s}$ on filter paper.

Stress treatments

Heat stress was given in an incubator (hot-air treatment, HAT) or in a hot-water bath (hot-water treatment, HWT). In the latter case, 20 seedlings were in an Eppendorf tube in $1 \mathrm{ml}$ liquid medium unless indicated otherwise. Drought stress was imposed in a sealed 60-mm Petri dish on top of blue silica gel. For salinity stress, seedlings, grown for 5 day at $20^{\circ} \mathrm{C}$, were exposed to nutrient medium with $600 \mathrm{mM} \mathrm{NaCl}$ for $4 \mathrm{~h}$ and then transferred back to standard medium.

Statistical analysis

Survival was scored 12 day after the stress. For each determination six Petri dishes with 20 seedlings each were used. In the figures and tables, means \pm SEs are shown.

\section{Results and discussion}

Development of test systems for drought and heat

For drought stress, various experimental setups may be chosen (Verslues et al. 2006). Plantlets may be cultured on medium with low water potential obtained by adding a high-molecular-weight solute such as polyethylene glycol 6000. Alternatively, plantlets may be air-dried. The latter may be done by taking the seedlings from the nutrient medium and leaving them on the laminar flow bench. Preferably, though, drought stress should be given under controlled conditions. In our experiments, seedlings were removed from the nutrient medium, kept constantly for $2 \frac{1}{2} \mathrm{~h}$ in a sealed $60-\mathrm{mm}$ Petri dish on filter paper on top of blue silica gel and then transferred back to the nutrient medium. Survival was scored 12 days after the stress. Only ca. $15 \%$ of the seedlings survived the drought-stress treatment. Detached leaves of Arabidopsis plants lose $50 \% \mathrm{FW}$ in $6 \mathrm{~h}$ 
(Verslues et al. 2006) whereas in-vitro grown Arabidopsis seedlings lose $50 \% \mathrm{FW}$ in $1 / 2 \mathrm{~h}$ of drought stress (de Klerk 2007). After $2 \frac{1}{2} \mathrm{~h}$, the seedlings have lost $85 \% \mathrm{FW}$. The rapid loss of FW of in-vitro grown seedlings is for the greater part likely due to the specific tissue culture conditions which strongly decrease the water retention capacity (de Klerk and Wijnhoven 2005).

Heat stress may be given simply by transferring Petri dishes into an incubator. This is referred to as "hot-air treatment" (HAT). However, because thermal conduction of air is poor, heat treatment may be applied in hot water in a small tube in a water bath or in a thermoblock. A hot-water treatment (HWT) was given by Larkindale and Knight (2002). We compared both methods. After a HWT far more seedlings died than after a HAT (Table 1).

Various differences between HWT and HAT may be responsible for the much higher level of damage during HWT. We assume that poor gas exchange leading to anaerobiosis is a major factor (Jackson 1985; Dolferus et al. 2003). To examine the effect of low oxygen, the number of seedlings per Eppendorf tube was increased so that per seedling even less oxygen would be available. Figure 1 shows that for tubes with five or ten seedlings survival was ca $40 \%$, but when the HWT was applied to tubes with 40 seedlings, survival was close to $0 \%$ (Fig. 1). We also added an oxygen donor, $\mathrm{H}_{2} \mathrm{O}_{2}$. This resulted in a somewhat increased survival (10\% to $20 \%$; data not shown). In horticulture, HWTs are being used routinely to render seeds, bulbs and fruits free from micro-organisms (Grondeau and Samson 1994). This effect of HWT is likely related to the synthesis of pathogenesis related proteins during the HWT (Van Loon et al. 2006).

Table 1 Survival of Arabidopsis seedlings after heat stress at $42.5^{\circ} \mathrm{C}$. Heat stress treatment was administered either to seedlings submerged in liquid medium $(\mathrm{HWT}=$ hot water treatment) or to seedlings kept on solidified medium in a Petri dish $($ HAT $=$ hot air treatment)

\begin{tabular}{lcc}
\hline & HWT & HAT \\
\hline $1 \mathrm{~h}$ & $0 \pm 0 \%$ & $100 \pm 0 \%$ \\
$2 \mathrm{~h}$ & $2.1 \pm 2.1 \%$ & $100 \pm 0 \%$ \\
$3 \mathrm{~h}$ & $0 \pm 0 \%$ & $90.1 \pm 3.2 \%$ \\
\hline
\end{tabular}

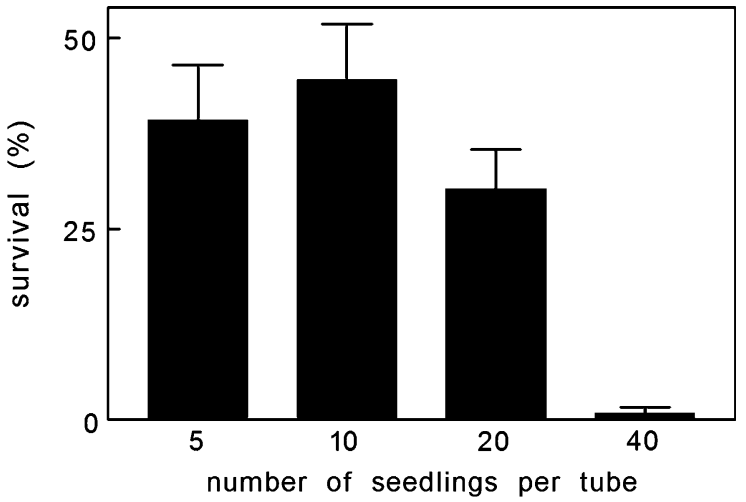

Fig. 1 Survival of Arabidopsis seedlings after a severe HWT of $2 \mathrm{~h}$ at $40^{\circ} \mathrm{C}$. The HWT was given in an Eppendorf tube in 1 $\mathrm{ml}$ liquid medium. The effect of the number of seedlings per tube was determined. Survival was scored 12 day after the stress

Addition of protectants and signal molecules

For protectants, we focused on polyamines and carbohydrates. The results with polyamines have been reported previously (de Klerk 2007). Putrescine increased survival after the $2 \frac{1}{2} \mathrm{~h}$ drought treatment from $15 \%$ (control) to $85 \%$ (treated for 2 day with 10 $\mathrm{mM}$ putrescine). At the same time, though, putrescine is toxic as shown by protracted culture in putrescinecontaining medium (de Klerk 2007).

With respect to protection by carbohydrates, until recently only few plant species, viz., desiccation tolerant 'resurrection plants' were thought to synthesize trehalose. However, most plant species accumulate sucrose during stress (Wingler 2002). Trehalose protects proteins and membranes by replacing water through hydrogen bonding to polar residues and is superior in protection because of its physical-chemical properties (Lerbret et al. 2005; Wingler 2002). We tested trehalose at a range of concentrations $(10,20,60$ and $100 \mathrm{mM})$ and found that the highest concentration tested (100 mM) resulted in high protection against drought $(80 \%$ survival, Fig. 2). At this concentration, protection by other carbohydrates is less (Fig. 3).

Protectants act by direct interaction with vulnerable macromolecules or by osmoprotection. Signaling molecules act in a very different way. They evoke a protective action by the tissue, among others the synthesis of protective molecules such as the aforementioned low-molecular-weight compounds but also proteins such as LEA-proteins, HSPs and enzymes to 

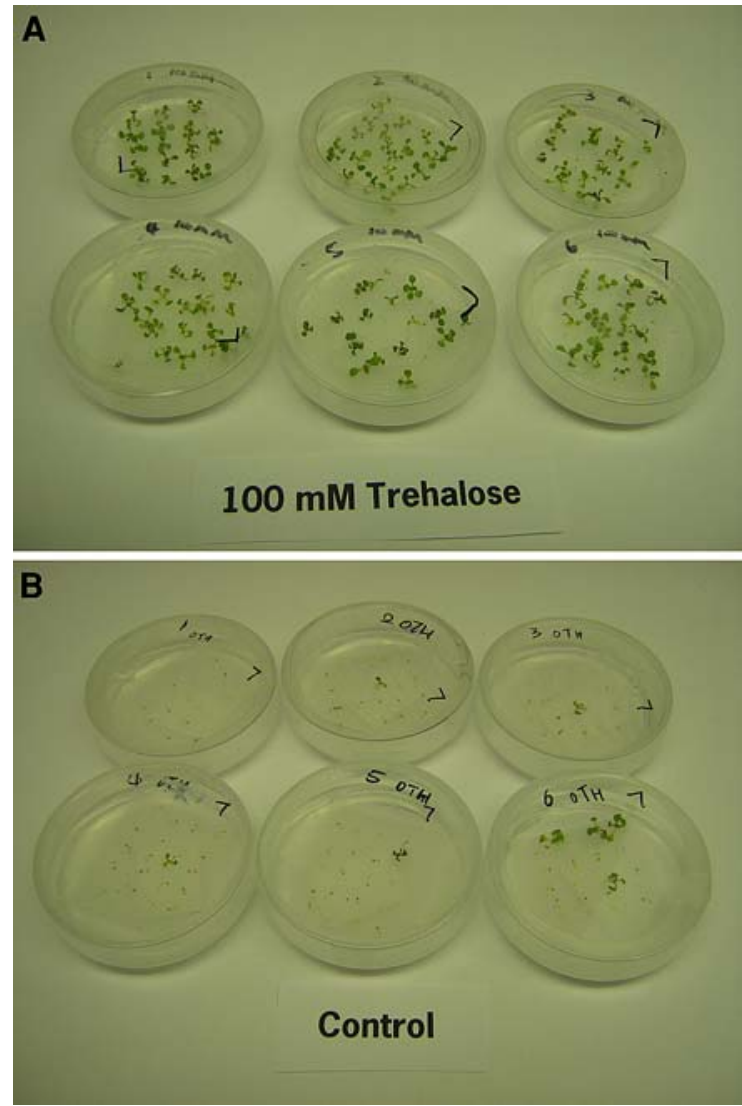

Fig. 2 Survival of Arabidopsis seedlings after severe drought stress. The seedlings were germinated for 3 day on standard medium and after that for 2 day on medium with $100 \mathrm{mM}$ trehalose (a) or on standard medium (b). Then they were drought-treated for $2 \frac{1}{2} \mathrm{~h}$ and transferred back to standard medium. The photo was taken 12 day after the stress

remove toxic active oxygen species. We have examined salicylic acid and acetylsalicylic acid and only found a minor protective effect of acetylsalicylic acid (results not shown). Various signaling molecules have been examined by other authors and significant improvements have been obtained with salicylic acid (in contrast to our results, Lopez-Delgado et al. 1998), abscisic acid (Gómez-Cadenas et al. 2003) and jasmonates (Wasternack 2007).

Hardening by a mild prestress

Mild heat pretreatments enhance thermotolerance (Burke 1998; Larkindale and Knight 2002). Drought hardening by mild drought treatments is well known in agriculture and forestry (Lemcoff et al. 2006; Villar-Salvador et al. 2004). When 5 day-old

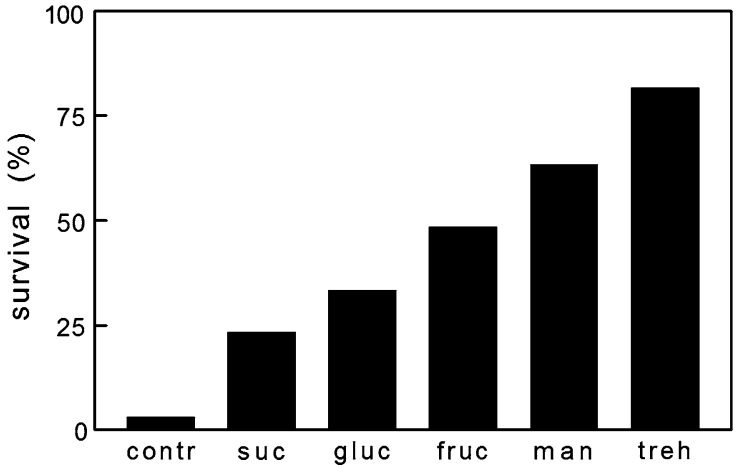

Fig. 3 Survival of Arabidopsis seedlings after severe drought stress. The seedlings were germinated for 3 day on standard medium and after that for 2 day on medium with $100 \mathrm{mM}$ sucrose, glucose, fructose, mannose or trehalose. $100 \mathrm{mM}$ was added in addition to $1.5 \%$ sucrose $(44 \mathrm{mM})$. Then they were drought-treated for $2 \frac{1}{2} \mathrm{~h}$ and transferred back to standard medium. Survival was scored 12 day after the stress

seedlings were pretreated with a mild HWT $\left(1 \frac{1 / 2}{h}\right.$ at $\left.35^{\circ} \mathrm{C}\right)$, survival after severe HWT $\left(2 \mathrm{~h}\right.$ at $\left.40^{\circ} \mathrm{C}\right)$ applied $4 \mathrm{~h}$ after the mild stress was increased from ca. $10 \%$ to ca $85 \%$. Since maximum protection was reached some hours after the mild stress, the development of stress resistance continued for some time after the mild stress. After that, both the development of stress-resistance and the stress resistance itself decreased rapidly. After $48 \mathrm{~h}$, only $50 \%$ of the maximal effect was still present (Fig. 4). Similar effects were obtained when a mild drought stress was

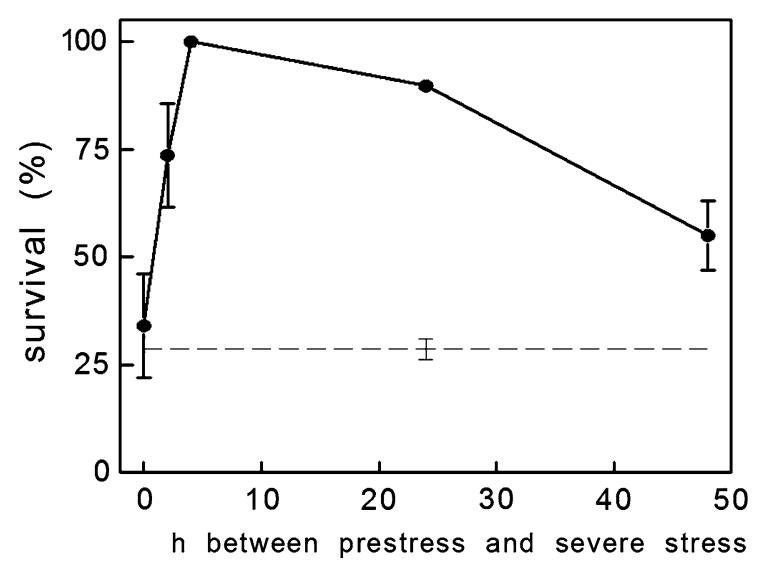

Fig. 4 Survival of Arabidopsis seedlings after a severe HWT. The seedlings were germinated for 5 day on standard medium followed by a pretreatment for $1 \frac{1}{2} \mathrm{~h}$ at $35^{\circ} \mathrm{C}$. They were kept at standard conditions for increasing periods of time $(0-48 \mathrm{~h})$ and then received a severe HWT of $2 \mathrm{~h}$ at $40^{\circ} \mathrm{C}$. Survival was scored 12 day after the stress 


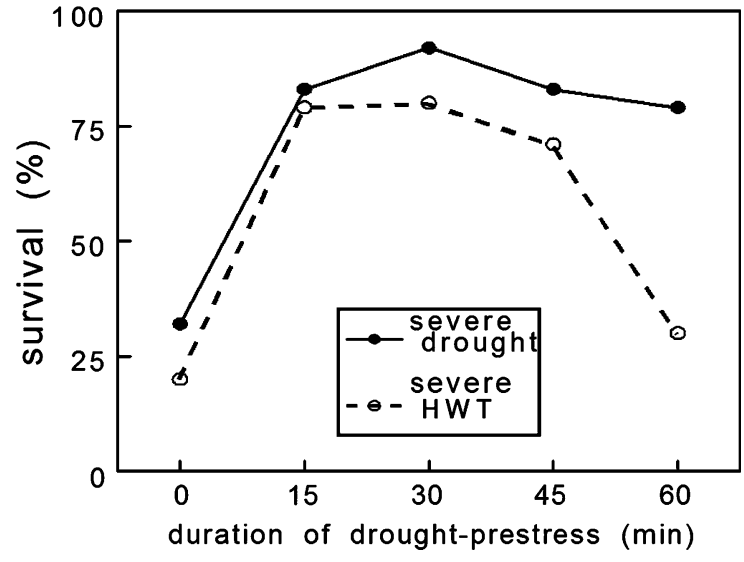

Fig. 5 Survival of Arabidopsis seedlings after a severe HWT or severe drought stress. The seedlings were germinated for 5 day on standard medium and then hardened by a drought treatment of increasing duration. After the drought pretreatment, the seedlings were reimbibed for $4 \mathrm{~h}$ under standard conditions. Then they received a severe drought treatment $\left(2 \frac{1}{2}\right.$ h drought) or a severe HWT $\left(2 \mathrm{~h}\right.$ at $\left.40^{\circ} \mathrm{C}\right)$. Survival was scored 12 day after the stress

given before the severe drought stress. When seedlings were dried under standard conditions for a short period (15-60 min), reimbibed for $4 \mathrm{~h}$ and then subjected to the $2 \frac{1 / 2}{\mathrm{~h}}$ drought stress, they showed strongly increased survival (Fig. 5). We examined cross-protection and found that brief exposure to drought stress protected against severe HWT (Fig. 5).
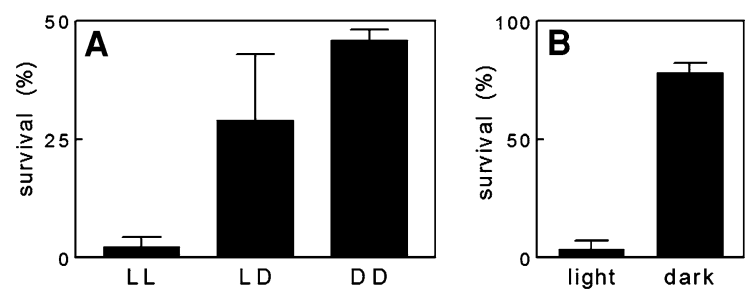

Fig. 6 Effect of darkness on survival after stress. (a) Seedlings were grown for 5 day in the dark or in the light. Then they received the severe drought stress. After that they were either kept in the light directly or after an intermediate period of 3 day darkness. Survival was scored 12 day after the stress. LL: Light-grown and cultured in the light directly after the stress. LD: Light-grown, cultured for 3 day in the dark after the stress and then in the light; DD: Dark-grown, cultured for 3 day in the dark after the stress and then in the light. (b) Seedlings were grown for 5 day under standard conditions, and then transferred for $4 \mathrm{~h}$ to medium with $600 \mathrm{mM} \mathrm{NaCl}$. After the salinity stress, they were transferred back to standard medium and either kept directly in the light or after an intermediate period of darkness of 3 day transferred to the light. Survival was scored 12 day after the stress
Darkness

Photosynthesis is a major source of activated oxygen. Larkindale and Knight (2002) and Larkindale et al. (2005) report that the heat damage to Arabidopsis seedlings is reduced by culture in dark. In agreement with this, we observed that dark-grown seedlings have a strongly increased tolerance to drought treatment (Fig. 6a). Figure 6b shows that in darkness, the sensitivity to salinity is also much reduced. Salt stress increases photodamage to PSII (Murata et al. 2007). Similar effects were obtained with apple shoots exposed to heat or salinity stress (data not shown).

\section{Conclusion}

The aim of this research was to examine the effect of various protective measures in vitro to overcome damage by abiotic stress. Several protective procedures have been identified using seedlings of Arabidopsis exposed to severe abiotic stresses. In tissue culture, various instances of abiotic stress occur (see Introduction) and future experiments concern measures protective towards these alleged stresses. Positive effects of protectants on performance during acclimatization have been reported previously (de Klerk 2002).

Acknowledgements We wish to thank Anne Bremkens, Guilhem Reyt, Marion Berardi and Pingping Huang for their excellent assistance and Frans Krens for reading the manuscript.

Open Access This article is distributed under the terms of the Creative Commons Attribution Noncommercial License which permits any noncommercial use, distribution, and reproduction in any medium, provided the original author(s) and source are credited.

\section{References}

Bae H, Herman E, Sicher R (2005) Exogenous trehalose promotes non-structural carbohydrate accumulation and induces chemical detoxification and stress response proteins in Arabidopsis thaliana grown in liquid culture. Plant Sci 168:1293-1301

Burke JJ (1998) Characterization of acquired thermotolerance in soybean seedlings. Plant Physiol Biochem 36:601-607

Chen C (2004) Humidity in plant tissue culture vessels. Biosyst Eng 88:231-241

de Klerk GJ (2002) Rooting of microcuttings: theory and practice. In Vitro Cell Dev Biol Plant 38:415-422 
de Klerk GJ (2007) Stress in plants cultured in vitro. Prop Ornam Plants 7:129-137

de Klerk GJ, Wijnhoven F (2005) Water retention capacity of tissue-cultured plants: performance of leaves from in vitro germinated mungbean seedlings. Prop Ornam Plants 5:14-18

Dolferus R, Klok EJ, Delessert C, Wilson S, Ismond KP, Good AG, Peacock WJ, Dennis ES (2003) Enhancing the anaerobic response. Ann Bot 91:111-117

Garg AK, Kim JK, Owens TG, Ranwala AP, Do Choi Y, Kochian LV, Wu RJ (2002) Trehalose accumulation in rice plants confers high tolerance levels to different abiotic stresses. Proc Natl Acad Sci USA 99:1589815903

Gómez-Cadenas A, Arbona V, Jacas J, Primo-Millo E, Talon M (2003) Abscisic acid reduces leaf abscission and increases salt tolerance in citrus plants. J Plant Growth Regul 21:234-240

Grondeau C, Samson R (1994) A review of thermotherapy to free plant materials from pathogens, especially seeds from bacteria. Crit Rev Plant Sci 13:57-75

Jackson MB (1985) Ethylene and responses of plants to soil waterlogging and submergence. Annu Rev Plant Physiol 36:145-174

Kasukabe Y, He L, Nada K, Misawa S, Ihara I, Tachibana S (2004) Overexpression of spermidine synthase enhances tolerance to multiple environmental stresses and up-regulates the expression of various stress-regulated genes in transgenic Arabidopsis thaliana. Plant Cell Physiol 45:712-722

Larkindale J, Knight MR (2002) Protection against heat stressinduced oxidative damage in Arabidopsis involves calcium, abscisic acid, ethylene, and salicylic acid. Plant Physiol 128:682-695

Larkindale J, Hall JD, Knight MR, Vierling E (2005) Heat stress phenotypes of Arabidopsis mutants implicate multiple signaling pathways in the acquisition of thermotolerance. Plant Physiol 138:882-897

Lemcoff JH, Ling F, Neumann PM (2006) Short episodes of water stress increase barley root resistance to radial shrinkage in a dehydrating environment. Physiol Plant 127:603-611

Lerbret A, Bordat P, Affouard F, Descamps M, Migliardo F (2005) How homogeneous are the trehalose, maltose, and sucrose water solutions? An insight from molecular dynamics simulations. J Phys Chem B 109:1104611057
Lopez-Delgado H, Dat JF, Foyer CH, Scott IM (1998) Induction of thermotolerance in potato microplants by acetylsalicylic acid and $\mathrm{H}_{2} \mathrm{O}_{2}$. J Exp Bot 49:713-720

Malik MK, Slovin JP, Hwang CH, Zimmerman JL (1999) Modified expression of a carrot small heat shock protein gene, Hsp17.7, results in increased or decreased thermotolerance. Plant J 20:89-99

McKersie BD, Bowley SR, Jones KS (1999) Winter survival of transgenic alfalfa overexpressing superoxide dismutase. Plant Physiol 119:839-847

Murashige T, Skoog F (1962) A revised medium for rapid growth and bio assays with tobacco tissue cultures. Physiol Plant 15:473-497

Murata N, Takahashi S, Nishiyama Y, Allakhverdiev SI (2007) Photoinhibition of photosystem II under environmental stress. Biochim Biophys Acta Bioenerg 1767:414-421

RoyChoudhury A, Roy C, Sengupta DN (2007) Transgenic tobacco plants overexpressing the heterologous lea gene Rab16A from rice during high salt and water deficit display enhanced tolerance to salinity stress. Plant Cell Rep 26:1839-1859

Sabehat A, Weiss D, Lurie S (1998) Heat-shock proteins and cross-tolerance in plants. Physiol Plant 103:437-441

Tunnacliffe A, Wise MJ (2007) The continuing conundrum of the LEA proteins. Naturwissenschaften 94:791-812

Van Loon LC, Rep M, Pieterse CMJ (2006) Significance of inducible defense-related proteins in infected plants. Annu Rev Phytopathol 44:135-162

Verslues PE, Agarwal M, Katiyar-Agarwal S, Zhu J, Zhu JK (2006) Methods and concepts in quantifying resistance to drought, salt and freezing, abiotic stresses that affect plant water status. Plant J 45:523-539

Villar-Salvador P, Planelles R, Oliet J, Peñuelas-Rubira JL, Jacobs DF, González M (2004) Drought tolerance and transplanting performance of holm oak (Quercus ilex) seedlings after drought hardening in the nursery. Tree Physiol 24:1147-1155

Wang W, Vinocur B, Altman A (2003) Plant responses to drought, salinity and extreme temperatures: towards genetic engineering for stress tolerance. Planta 218:1-14

Wasternack C (2007) Jasmonates: an update on biosynthesis, signal transduction and action in plant stress response, growth and development. Ann Bot 100:681-697

Wingler A (2002) The function of trehalose biosynthesis in plants. Phytochemistry 60:437-440

Zeid IM, Shedeed ZA (2006) Response of alfalfa to putrescine treatment under drought stress. Biol Plant 50:635-640 